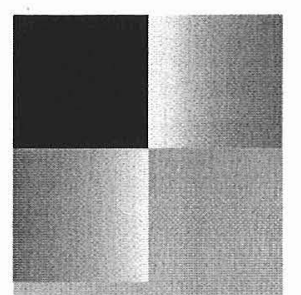

\title{
Increasing Access to Cardiac Rehabilitation
}

\author{
AUTOMATIC REFERRAL TO THE PROGRAM \\ NEAREST HOME
}

Sherry L. Grace, PhD; Alexandra Evindar, MS; Tabitha Kung, BSc; Patricia Scholey, BN, RN; Donna E. Stewart, MD, FRCPC

Substantial health risks exist after coronary events and procedures, ${ }^{1}$ and cardiac rehabilitation (CR) plays a large role in reducing these risks. ${ }^{2}$ However, most research demonstrates low use of $\mathrm{CR}$ and differential referral patterns.3,4 The directions for future research outlined in much of the CR literature promote automatic referral to provide consistent referral based on patient need.5,6 However, to the authors' knowledge, this type of referral intervention has not been systematically defined, implemented, or evaluated in the literature.

The primary objective of the current study was to compare $\mathrm{CR}$ participation after automatic electronic referral to a site nearest home with usual referral. The secondary objective was to examine differences in $\mathrm{CR}$ participation based on sex and indication for referral.

\section{METHODS}

The Trillium Health Centre (THC) Cardiac Wellness and Rehabilitation Centre in Toronto, Ontario, has instituted automatic referral. The THC is a large, urban tertiary care facility. Cardiac rehabilitation is provided at no or minimal cost to cardiac patients within the universal healthcare system. Hospital electronic patient records are used to prompt the standard order of a CR referral for all eligible cardiac patients (based on clinical practice guidelines). 7,8 An information package including a personalized letter stating the name of the referring physician, a program brochure, and a request that the patient call to book an appointment is mailed to the patient's home. Patients who live outside the geo- graphic also are sent a similar package, but provided with the contact information for the site nearest their home. This alternate site also is sent the patient's contact information.

This study used a cross-sectional comparative design. Data were obtained through access to the cardiac patient database compiled between April 26, 2001 and May 15, $2002(\mathbf{n}=1611)$. The database was screened for all the patients with atherosclerotic heart disease. This screening yielded a set of 1501 cases, from which a random sample of 501 patients was extracted for initial contact. Of these 501 patients with atherosclerotic heart disease, 367 were males (73.3\%) and 134 were females $(26.7 \%)$. The most frequent referral event was coronary artery bypass graft surgery (CABG; $n=296$, $60.5 \%$ ), followed by percutaneous coronary intervention $(n=85,17.4 \%)$, myocardial infarction $(n=69$, $14.1 \%)$, and coronary artery disease $(n=51,10.2 \%)$ more generally. The mean number of months from the referral event to study participation was $15.31 \pm 3.66$ ).

The THC chart data included gender, indication for referral $(\mathrm{CABG}$, percutaneous coronary intervention, myocardial infarction, coronary artery disease), date of 


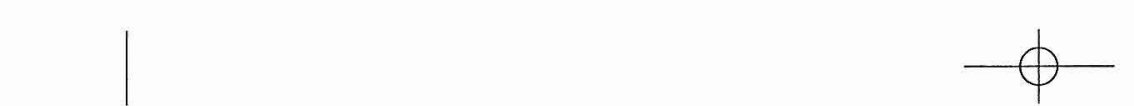

Table 1 - CONTINGENCY TABLE AND CHI-SOUARE ANALYSIS COMPARING CR PARTICIPATION AFTER AUTOMATIC REFERRAL VERSUS CR PILOT PROJECT DATA FOR THE PROVINCE*

\begin{tabular}{lccc}
\hline & Participated in CR & Did not Participate in CR & \% Participating \\
\hline Automatic referral-THC & 215 & 286 & $42.9 \% \dagger$ \\
Usual referral-Province & 9796 & 35581 & $21.6 \%$ \\
\hline
\end{tabular}

CR, cardiac rehabilitation; THC, Trillium Health Center.

*The THC participation data are based on the most conservative estimate assuming that nonverifiable patients did not attend CR.

t\% participating is significantly greater, $P<.005$.

referral event, and site to which the patient was referred. The $22 \mathrm{CR}$ sites "nearer home" to which patients were referred were contacted by mail to obtain CR participation data (100\% response rate). Cardiac rehabilitation site chart data included whether the patients declined to participate, whether they partially or fully completed the program, and the date of $\mathrm{CR}$ graduation.

The CR participation rates after automatic referral were compared with those after usual referral. Recently, the provincial government funded a pilot project that examined the CR service supply and the need across the province from January 1, 2001 through March 31, 2002.9 The CR protocol was standardized according to international clinical practice guidelines.10,11 The participants included 24 CR sites (THC included) across seven regions and 4477 cardiac patients. Usual referral consisted of sitespecific referral practices initiated by primary care physicians, cardiologists, or cardiovascular surgeons, and, in some cases, self-referral. In this pilot project, the eligible $\mathrm{CR}$ referral population was computed on the basis of hospital discharge abstracts (from the Canadian Institutes of Health Information) and mapped using Geographical Information Systems software to determine catchment area cardiac discharges. According to these calculations, approximately $22 \%$ of eligible cardiac patients participated in $\mathrm{CR}$ during the pilot project. The region in which THC is located includes six other CR sites, and the region had $16 \% \mathrm{CR}$ participation in the pilot project.

The frequency of referral completion, patient participation, and site attendance were examined. Participation rates from the CR pilot project ${ }^{9}$ for the province and region were abstracted, and $2 \times 2$ contingency tables were created to compute $\chi^{2}$ tests of significance compared with participation rates in the current sample. Pearson's $\chi^{2}$ also was used to test for significant differences in $\mathrm{CR}$ participation based on sex and referral event.

\section{RESULTS}

According to the random sample of 501 patients with atherosclerotic heart disease from the THC chart data, $323(64.5 \%)$ were referred to the THC, $145(28.9 \%)$ were referred to a site nearer home, and $33(6.6 \%)$ were ineligible for CR and not referred (because of decease or a comorbid condition that precluded participation). The 145 nonlocal patients were referred to 22 alternative sites nearer home.

After referral, 196 (39.1\%) patients attended CR at the site to which they were originally referred, and $15(3.0 \%)$ attended at an alternate CR site. Whereas 73 (14.6\%) declined to participate in CR at the THC, 95 (19.0\%) simply did not reply. Of the patients referred to CR sites nearer home, $56(11.2 \%)$ declined to participate, but there was no record for 12 patients $(2.4 \%)$ at the site nearer home. In addition, 37 (7.4\%) patients were medically ineligible or deceased, and $17(3.4 \%)$ were unverifiable at any site.

Verified participation included 130 patients (25.9\%) at the THC, and 85 at a site nearer home (17\%). Almost half of the sample ( $\mathrm{n}=235,46.9 \%$ ) did not attend CR; 34 (6.8\%) were medically ineligible; and 17 (3.4\%) were unverifiable. As compared with the CR pilot project participation rates, automatic referral resulted in significantly greater CR participation than usual referral in the province and in the region (Tables 1 and 2, respectively). Of the 215 verified CR participants, 184 (85.6\%) achieved their goals and graduated, 23 (10.7\%) attended some sessions but did not graduate, and $1(0.5 \%)$ was still participating. For 7 participants $(3.3 \%)$, this information was not available.

Differences in participation based on gender and indication for referral also were examined. Of the 215 CR participants, 171 (50.6\%) were male and 44 (39.3\%) were female. According to $\chi^{2}$ analysis, there was no significant difference in $\mathrm{CR}$ use based on referral event (132 CABG participants, 61.4\%; 154 CABG nonparticipants, $\left.61.1 \% ; \chi^{2}[1]=0.16\right)$, but women were more often nonparticipants $(\mathrm{n}=74,29.4 \%)$ than participants (n $=44,20.5 \% ; \chi^{2}[1]=4.87 ; P=.03$ ).

\section{DISCUSSION}

Cardiac rehabilitation has demonstrated beneficial effects on morbidity and mortality. ${ }^{2}$ Therefore, it is essential to maximize participation. However, referral to 


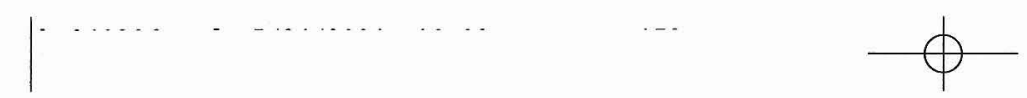

\begin{tabular}{|c|c|c|c|}
\hline & Participated in CR & Did not Participate in CR & $\%$ Participating \\
\hline Automatic referral-THC & 215 & 286 & $42.9 \% *$ \\
\hline Usual referral—Region & 1448 & 2682 & $16.1 \%$ \\
\hline
\end{tabular}

CR, cardiac rehabilitation. THC, Trillium Health Center.

*\% participating is significantly greater, $P<.025$.

CR is inconsistent. 12 In this study, automatic referral resulted in CR participation among $43 \%$ of the eligible patients. This represents an improvement over the $15 \%$ to $20 \%$ participation rates commonly reported in the literature, ${ }^{6}$ and a significant increase in comparison with provincial (22\%) and regional (16\%) participation. 9

The automatic referral process resulted not only in increased program entry, but also in consistent participation regardless of the indication for referral. Contrary to previous findings, ${ }^{4}$ there were no differences in CR participation based on a CABG, percutaneous coronary intervention, myocardial infarction, or coronary artery disease diagnosis. Although clinical practice guidelines vary in terms of indications for referral, the scientific literature substantiates the benefits of CR for each of these diagnoses.8,10,11 However, gender differences persisted, with female cardiac patients failing to complete $\mathrm{CR}$ more often than males (although 39.3\% of eligible females participated in $\mathrm{CR}$, which is higher than the rates reported in the literature13). This difference could be attributable to the perception of $\mathrm{CR}$ as male oriented.14 Moreover, women cardiac patients often are older, 13 have a greater number of comorbid conditions, and face more transportation barriers, 13 all factors that may affect the desirability of $\mathrm{CR}$.

Automated communication between healthcare departments has been shown to increase continuity of care and reduce time required for referral. 15 At communication with the $22 \mathrm{CR}$ sites nearer home, some disruption in the referral process was noted. True automatic referral to sites nearest home would require synergy of information systems between sites. A trial of regionalized automatic referral may be viable in the managed care environment.

The strengths of this study included the random sampling of CR eligible patients who attended multiple CR sites and the verification of participation at these sites by chart audit. The limitations included the retrospective nature of the study and the reliance upon comparative data from the Cardiac Care Network (CCN) report. However, data for both this study and the $\mathrm{CCN}$ report are based on the same guideline-based CR model, are collated from the same historical period, and allow for an epidemiologic snapshot of access. Future research is needed for prospective examination of automatic referral, as compared with usual referral, to determine whether these findings are replicable. A final limitation relates to the operationalization of automatic referral, whereby patients were mailed an information packet, but were not provided an intake appointment unless they telephoned the CR program. When the THC first instituted automatic referral, the staff booked intake appointments for all referrals. They quickly found their waiting list lengthened, and some patients did not attend. Future research is required to compare different automatic referral processes, and to disentangle the effects of referral itself from patient completion of the referral.

In conclusion, to the authors' knowledge, this is the first study to examine empirically the effect of automatic referral on CR utilization. Automatic referral resulted in greater CR participation ( $46 \%$ ) than observed in the literature $(15-20 \%)$, the province $(22 \%)$, and the surrounding region (16\%). These data also demonstrate that when eligible cardiac patients are referred universally to CR on the basis of need, no variation in utilization based on type of referral event is observed. The use of automatic referral has the potential not only to increase utilization, but also to reduce disparities in access.

\section{Acknowledgments}

The authors gratefully acknowledge the CIHR Institute of Gender and Health for a postdoctoral fellowship to S. Grace, which supported this study.

\section{References}

1. Law MR, Watt HC, Wald NJ. The underlying risk of death after myocardial infarction in the absence of treatment. Arch Intern Med. 2002;162:2405-2410.

2. Jolliffe JA, Rees K, Taylor RS, Thompson D, Oldridge N, Ebrahim S. Exercise-based rehabilitation for coronary heart disease. Cocbrane Database Syst Rev. 2001(4).

3. Grace SL, Abbey S, Shnek Z, Irvine J, Franche RL, Stewart D. Cardiac rehabilitation II: Referral and participation. Gen Hosp Psycb. 2002;24:127-134.

4. Barber K, Stommel M, Kroll J, Holmes-Rovner M, McIntosh B. Cardiac rehabilitation for community-based patients with myocardial infarction: factors predicting discharge recommendation and participation. J Clin Epidemiol. 2001;54:1025-1030.

5. King KM, Teo KK. Cardiac rehabilitation referral and attendance: not one and the same. Rehabil Nurs. 1998;23:246-251.

6. Daly J, Sindone AP, Thompson DR, Hancock K, Chang E, Davidson P. Barriers to participation in and adherence to cardiac 
rehabilitation programs: a critical literature review. Prog Cardiovasc Nurs. 2002;17:8-17.

7. Canadian Association of Cardiac Rehabilitation. Canadian Guidelines for Cardiac Rebabilitation and Cardiovascular Disease Prevention. 1st ed. Winnipeg: CACR; 1999.

8. American Association of Cardiovascular and Pulmonary Rehabilitation. Guidelines for Cardiac Rebabilitation and Secondary Prevention Programs. 4th ed. Champaign, Ill: Human Kinetics; 2004.

9. Cardiac Care Network. The Ontario cardiac rebabilitation pilot project: report and recommendations. Toronto, Ontario; Available at http://www.ccn.on.ca/rehabpublic/FinalReport_PDF_Sept30_02_ FINALpassword.pdf. Accessed May 2004.

10. North of England Evidence-based Guidelines Development Project. Primary care management of secondary propbylaxis for patients who bave experienced a myocardial infarction: drug treatment, cardiac rebabilitation and dietary manipulation. 2001. Centre for Health Services Research University of Newcastle Upon Tyne. Available at: www.nelh.nhs.uk.
11. Goble AJ, Worcester MUC. Best Practice Guidelines for Cardiac Rehabilitation and Secondary Prevention. Victoria, Australia: Department of Human Services Victoria; 1999.

12. Blackburn GG, Foody JM, Sprecher DL, Park E, Apperson-Hansen C, Pashkow FJ. Cardiac rehabilitation participation patterns in a large, tertiary care center: evidence for selection bias. $J$ Cardiopulm Rehabil. 2000;20:189-195.

13. Thomas R, Miller N, Lamendola $\mathrm{C}$, et al. National survey of gender differences in cardiac rehabilitation programs. $J$ Cardiopulm Rebabil. 1996;16:402-412.

14. Benz Scott LA, Ben-Or K, Allen JK. Why are women missing from outpatient cardiac rehabilitation programs? A review of multilevel factors affecting referral, enrollment, and completion. $J$ Women's Health. 2002;11:773-791.

15. Crosswhite R, Beckham SH, Gray P, Hawkins PR, Hughes J. Using a multidisciplinary automated discharge summary process to improve information management across the system. Am J Manag Care. 1997:3:473-479. 